Article

\title{
Regularity in Topological Modules
}

\author{
Francisco Javier Garcia-Pacheco $D$ \\ Department of Mathematics, College of Engineering, University of Cadiz, 11519 Puerto Real, Spain; \\ garcia.pacheco@uca.es
}

Received: 20 July 2020; Accepted: 9 September 2020; Published: 13 September 2020

\begin{abstract}
The framework of Functional Analysis is the theory of topological vector spaces over the real or complex field. The natural generalization of these objects are the topological modules over topological rings. Weakening the classical Functional Analysis results towards the scope of topological modules is a relatively new trend that has enriched the literature of Functional Analysis with deeper classical results as well as with pathological phenomena. Following this trend, it has been recently proved that every real or complex Hausdorff locally convex topological vector space with dimension greater than or equal to 2 has a balanced and absorbing subset with empty interior. Here we propose an extension of this result to topological modules over topological rings. A sufficient condition is provided to accomplish this extension. This sufficient condition is a new property in topological module theory called strong open property. On the other hand, topological regularity of closed balls and open balls in real or complex normed spaces is a trivial fact. Sufficient conditions, related to the strong open property, are provided on seminormed modules over an absolutely semivalued ring for closed balls to be regular closed and open balls to be regular open. These sufficient conditions are in fact characterizations when the seminormed module is the absolutely semivalued ring. These characterizations allow the provision of more examples of closed-unit neighborhoods of zero. Consequently, the closed-unit ball of any unital real Banach algebra is proved to be a closed-unit zero-neighborhood. We finally transport all these results to topological modules over topological rings to obtain nontrivial regular closed and regular open neighborhoods of zero. In particular, if $M$ is a topological $R$-module and $m^{*} \in M^{*}$ is a continuous linear functional on $M$ which is open as a map between topological spaces, then $\left(m^{*}\right)^{-1}(\operatorname{int}(B))$ is regular open and $\left(m^{*}\right)^{-1}(B)$ is regular closed, for $B$ any closed-unit zero-neighborhood in $R$.
\end{abstract}

Keywords: topological module; topological ring; normed module; absolutely valued ring; regular closed set; regular open set; closed-unit neighborhood of zero

MSC: 46H25; 16W80; 54H13

\section{Introduction}

Weakening the classical Functional Analysis results from real or complex normed spaces to real or complex topological vector spaces is an old trend that originally started with the study of the $w$-and $w^{*}$-topologies [1]. Over many years, the Functional Analysts observed that whereas many classical deep results for normed spaces also worked well for topological vector spaces, other results did not work as well and thus turned into pathological phenomena [2-4]. These pathological phenomena occur more often when the vector topologies are not Hausdorff [5].

The above mentioned trend evolved to a new research line consisting of generalizing, as much as possible, the classical results on real or complex topological vector spaces to the scope of topological vector spaces over division rings [6] or even topological modules over topological rings $[7,8]$. This evolution has considerably enriched the literature of Functional Analysis with 
deeper classical results as well as with pathological phenomena, as can be checked, for instance, in [9]. The books [10-13] are excellent references containing all the basics of the Functional Analysis literature in the topological module setting.

Several years ago, in ([14] (Theorem 3.2)), it was proved that every real or complex separable normed space with dimension greater than or equal to 2 has a balanced and absorbing subset with empty interior. Some time later, in ([15] (Theorem 1.1)), it was shown that every real or complex Hausdorff locally convex topological vector space with dimension greater than or equal to 2 has a balanced and absorbing subset with empty interior. This result motivated the study of the existence, in topological modules, of balanced and absorbing subsets which are not zero-neighborhoods. However, it was first necessary to extend the concept of balancedness and absorbance to the scope of topological modules. In [10], one can find these two concepts for topological vector spaces over absolutely valued division rings. In ([16] (Definition 2.1)), balanced and absorbing sets were defined for modules over topological rings. For this, a fundamental notion was necessary to introduce: closed-unit neighborhood of zero [16-18].

More recently, the isometric representations of groups on complex Banach spaces [19,20] motivated the study of isometric representations of groups on normed modules [21] (recall that an isometric group-representation is a group morphism between a group and the group of surjective linear isometries on a normed module over an absolutely valued ring). Later, the theory of Schauder bases has been redeveloped for topological modules in [22]. These two extensions generated the necessity of an operator theory for seminormed modules over absolutely semivalued rings. This new operator theory can be found in [23] and extends very accurately the classical operator theory on complex normed spaces [24]. The latest steps on this ongoing trend have been provided in two submitted preprints $[25,26]$. In the first one, the classical spectral theory is carried out to the scope of unitary algebras, where it is proved that the classical spectral decomposition in unitary complex Banach algebras also holds in general unitary algebras. In the second preprint, the locally convex topologies on complex topological vector spaces are transported to the scope of topological modules over topological rings. This is possible by introducing a new definition of convexity for modules different from the $C^{*}$-convexity [27] and that coincides with the usual concept of convexity when the topological ring is the real field. This new conception of convexity is known as $B^{+}$-convexity and it relies on the brand-new concept of closed-unit segment.

This paper is generally aimed at continuing the process of weakening the classical Functional Analysis results to the more general setting of topological modules. In particular, we pretend to extend two deep Functional Analysis results to topological modules:

1. Topological regularity of certain zero-neighborhoods such as closed balls, open balls and preimages of closed-unit zero-neighborhoods by continuous functionals (proved for real or complex normed spaces scattered throughout the literature [28]).

2. Existence of balanced and absorbing subsets which are not zero-neighborhoods (proved first in ([14] (Theorem 3.2)) for separable real or complex normed spaces with dimension greater than or equal to 2, and then in ([15] (Theorem 1.1)) for real or complex Hausdorff locally convex topological vector spaces with dimension greater than or equal to 2 ).

In both extensions, a new property on topological module theory is introduced: strong open property. This property is trivially verified by all real or complex topological vector spaces (in particular, by all real or complex normed spaces). Throughout this manuscript, we will see that not all the extensions are always possible, producing then the existence of pathological phenomena.

At the very beginning of every section, we will introduce all the necessary tools to prove our results. Here, in the rest of this introductory section, we will recall several basic concepts. Important topological notions and notations that will be used follow now. Let $X$ be a topological space. If $A \subseteq X$, then $\operatorname{int}(A), \mathrm{cl}(A), \mathrm{bd}(A)$ denote the interior, the closure, the boundary of $A$, respectively. For every $x \in X$, we will let $\mathcal{N}_{x}(X)$ denote the filter of neighborhoods of $x$. A subset $U \subseteq X$ is said to be regular 
open if $U=\operatorname{int}(\operatorname{cl}(U))$. A subset $B \subseteq X$ is said to be regular closed if $B=\operatorname{cl}(\operatorname{int}(B))$. Notice that if $U \subseteq X$ is open, then $\operatorname{cl}(U)$ is regular closed, and if $B \subseteq X$ is closed, then $\operatorname{int}(B)$ is regular open.

The following characterization of module topology ([10] (Theorem 3.6)) will be very much employed throughout this manuscript.

Theorem 1. Let $R$ be a topological ring and $M$ a topological $R$-module. If $\mathcal{B}$ is a basis of 0 -neighborhoods in $M$, then:

1. For each $W \in \mathcal{B}$ there is $U \in \mathcal{B}$ with $U+U \subseteq W$.

2. For each $W \in \mathcal{B}$ there is $U \in \mathcal{B}$ with $-U \subseteq W$.

3. For each $W \in \mathcal{B}$ there are $U \in \mathcal{B}$ and $V \in \mathcal{N}_{0}(R)$ with $U V \subseteq W$.

4. For each $W \in \mathcal{B}$ and each $r \in R$ there is $U \in \mathcal{B}$ with $r U \subseteq W$.

5. For each $W \in \mathcal{B}$ and each $m \in M$ there is $U \in \mathcal{N}_{0}(R)$ with $U m \subseteq W$.

Conversely, if $\mathcal{B}$ is a filter base on an $R$-module $M$ satisfying the previous properties, then there exists a unique module topology on $M$ in such a way that $\mathcal{B}$ is a basis of 0-neighborhoods.

Topological rings in which multiplicative inversion is continuous will be called inversiontopological rings. Notice that topological division rings are inversion-topological rings by definition. However, division topological rings need not be inversion-topological rings.

Let $R$ be a ring and $s \in R$. The sets of left-divisors and right-divisors of $s$ are defined and denoted by

$$
\ell d(s):=\{r \in R: \text { there exists } t \in R \backslash\{0\} \text { with } r t=s\}
$$

and

$$
r d(s):=\{r \in R: \text { there exists } t \in R \backslash\{0\} \text { with } t r=s\},
$$

respectively. The multiplicative group of invertibles in $R$ will be denoted, as usual, by $\mathcal{U}(R)$. Notice that $\mathcal{U}(R)=\ell d(1) \cap r d(1)$. On the other hand, $\ell d(0) \cap r d(1)=\varnothing=r d(0) \cap \ell d(1)$ and thus $(\ell d(0) \cup r d(0)) \cap \mathcal{U}(R)=\varnothing$. The ring $R$ is called an integral domain if $\ell d(0)=r d(0)=\{0\}$.

An $R$-module $M$ is called torsionfree provided that the equality $r m=0$ implies that either $m=0$ or $r \in \ell d(0) \cup r d(0)$. Suppose that $M$ is a topological $R$-module, we say that $M$ is strongly torsionfree if whenever $\left(r_{i} m\right)_{i \in I} \rightarrow 0$, then either $m=0$ or $\left(r_{i}\right)_{i \in I}$ is convergent to a zero divisor. In ([23] (Section 2.4)), a wider perspective on separation properties is given.

Keep in mind that the difference between a ring seminorm $\|\cdot\|$ and an absolute semivalue $|\cdot|$ is that the ring seminorm is submultiplicative $(\|r s\| \leq\|r\|\|s\|)$ and the absolute semivalue is multiplicative $(|r s|=|r||s|)$. Then a seminorm on an $R$-module $M$ is asked to be absolutely homogeneous $(\|r m\|=|r|\|m\|)$ if $R$ is absolutely semivalued, and submultiplicative $(\|r m\| \leq\|r\|\|m\|)$ if $R$ is seminormed. Throughout this paper, we will consider all module seminorms, ring seminorms and absolute semivalues to be nonzero.

If $X$ is a pseudometric space, then $\mathrm{B}_{X}(x, \delta)$ stand for the closed ball centered at $x \in X$ with radius $\delta>0$. The corresponding open ball is denoted by $\mathrm{U}_{X}(x, \delta)$, and the sphere centered at $x \in X$ with radius $\delta>0$ is denoted as $S_{X}(x, \delta)$. When $M$ is a seminormed module, then the closed-unit ball of $M$ is $\mathrm{B}_{M}:=\mathrm{B}_{M}(0,1)$, the open unit ball of $M$ is $\mathrm{U}_{M}:=\mathrm{U}_{M}(0,1)$ and the unit sphere is $\mathrm{S}_{M}:=\mathrm{S}_{M}(0,1)$.

\section{Regularity of Balls in Seminormed Modules}

Let $X$ be a pseudometric space. Since $\mathrm{U}_{X}(x, \delta)$ is open and $\mathrm{B}_{X}(x, \delta)$ and $\mathrm{S}_{X}(x, \delta)$ are closed for every $x \in X$ and every $\delta>0$, as a consequence, $\mathrm{U}_{X}(x, \delta) \subseteq \operatorname{int}\left(\mathrm{B}_{X}(x, \delta)\right)$ and $\mathrm{cl}\left(\mathrm{U}_{X}(x, \delta)\right) \subseteq \mathrm{B}_{X}(x, \delta)$. Notice that if $\mathrm{U}_{X}(x, \delta)=\operatorname{int}\left(\mathrm{B}_{X}(x, \delta)\right)$, then $\mathrm{U}_{X}(x, \delta)$ is regular open, and if $\mathrm{cl}\left(\mathrm{U}_{X}(x, \delta)\right)=\mathrm{B}_{X}(x, \delta)$, then $\mathrm{B}_{X}(x, \delta)$ is regular closed. Observe also that in any of the previous two cases, int $\left(\mathrm{S}_{X}(x, \delta)\right)=\varnothing$. The following examples show that $\mathrm{U}_{X}(x, \delta)=\operatorname{int}\left(\mathrm{B}_{X}(x, \delta)\right)$ and $\mathrm{cl}\left(\mathrm{U}_{X}(x, \delta)\right)=\mathrm{B}_{X}(x, \delta)$ are independent conditions in the sense that none of them implies the other. 
Example 1. Take $X:=[-1,1], x:=0$ and $\delta:=1$. Then

$$
\mathrm{cl}\left(\mathrm{U}_{X}(x, \delta)\right)=\operatorname{cl}((-1,1))=[-1,1]=\mathrm{B}_{X}(x, \delta)
$$

but

$$
\operatorname{int}\left(\mathrm{B}_{X}(x, \delta)\right)=\operatorname{int}([-1,1])=[-1,1] \neq(-1,1)=\mathrm{U}_{X}(x, \delta) .
$$

Example 2. Take $X:=\mathbb{R} \backslash(1,2), x:=0$ and $\delta:=2$. Then

$$
\operatorname{int}\left(\mathrm{B}_{X}(x, \delta)\right)=\operatorname{int}([-2,1] \cup\{2\})=(-2,1]=\mathrm{U}_{X}(x, \delta)
$$

but

$$
\operatorname{cl}\left(\mathrm{U}_{X}(x, \delta)\right)=\operatorname{cl}((-2,1])=[-2,1] \neq[-2,1] \cup\{2\}=\mathrm{B}_{X}(x, \delta) .
$$

Theorem 2. Let $R$ be a seminormed ring and $M$ a seminormed $R$-module. If $1 \in \mathrm{cl}\left(\mathrm{U}_{R}\right)$, then $\mathrm{cl}\left(\mathrm{U}_{M}(m, \delta)\right)=\mathrm{B}_{M}(m, \delta)$, and thus $\mathrm{B}_{M}(m, \delta)$ is regular closed, for every $m \in M$ and every $\delta>0$.

Proof. Fix an arbitrary $p \in \mathrm{S}_{M}(m, \delta)$. Let $\left(u_{n}\right)_{n \in \mathbb{N}} \subseteq \mathrm{U}_{R}$ be convergent to 1 . Then $\left(m+u_{n}(p-m)\right)_{n \in \mathbb{N}}$ is a sequence in $\mathrm{U}_{R}(m, \delta)$ which is convergent to $p$. As a consequence, $p \in \operatorname{cl}\left(\mathrm{U}_{M}(m, \delta)\right)$. The arbitrariness of $p$ shows that $\mathrm{cl}\left(\mathrm{U}_{M}(m, \delta)\right)=\mathrm{B}_{M}(m, \delta)$.

Please note that $1 \in \operatorname{cl}\left(\mathrm{U}_{R}\right)$ implies that $R$ is a unital seminormed ring, i.e., $\|1\|=1$.

Corollary 1. Let $R$ be a unital seminormed ring. The following conditions are equivalent:

1. $\mathrm{cl}\left(\mathrm{U}_{R}(r, \delta)\right)=\mathrm{B}_{R}(r, \delta)$ for all $r \in R$ and all $\delta>0$.

2. $\quad \mathrm{cl}\left(\mathrm{U}_{R}\right)=\mathrm{B}_{R}$.

3. $1 \in \mathrm{cl}\left(\mathrm{U}_{R}\right)$.

In this situation, $\mathrm{B}_{R}(r, \delta)$ is regular closed for all $r \in R$ and all $\delta>0$.

Proof. It is trivial that $(1) \Rightarrow(2)$. In order to prove that $(2) \Rightarrow(3)$, it only suffices to notice that $1 \in \mathrm{B}_{R}=\mathrm{cl}\left(\mathrm{U}_{R}\right)$ because $\|1\|=1$ by hypothesis. Finally, (3) $\Rightarrow(1)$ is a direct consequence of Theorem 2.

Since $|1|=1$ for any (nonzero) absolutely semivalued ring $R$, we have the following scholium.

Scholium 3. The following are equivalent for an absolutely semivalued ring $R$ :

1. $\quad \mathrm{cl}\left(\mathrm{U}_{R}(r, \delta)\right)=\mathrm{B}_{R}(r, \delta)$ for all $r \in R$ and all $\delta>0$.

2. $\quad \mathrm{cl}\left(\mathrm{U}_{R}\right)=\mathrm{B}_{R}$.

3. $1 \in \mathrm{cl}\left(\mathrm{U}_{R}\right)$.

In this situation, $\mathrm{B}_{R}(r, \delta)$ is regular closed for all $r \in R$ and all $\delta>0$.

Definition 1. Let $R$ be a seminormed ring and $M$ a seminormed $R$-module. It is said that $M$ satisfies the (strong) open property if for each open subset $U \subseteq M$ and each $m \in U$ there is an (invertible) element $s \in R$ with $\|s\|>1$ such that $s m \in U$.

We say that a seminormed ring $R$ satisfies the (strong) open property if it does as a left $R$-module. The open property serves as a sufficient condition for open balls to be dense in closed balls.

Theorem 4. Let $R$ be an absolutely semivalued ring and $M$ a seminormed $R$-module. If $M$ satisfies the open property, then $\mathrm{U}_{M}(m, \delta)=\operatorname{int}\left(\mathrm{B}_{M}(m, \delta)\right)$, and thus $\mathrm{U}_{M}(m, \delta)$ is regular open, for every $m \in M$ and every $\delta>0$. 
Proof. Suppose on the contrary that $\mathrm{U}_{R}(m, \delta) \subsetneq \operatorname{int}\left(\mathrm{B}_{M}(m, \delta)\right)$. Then there exists $n \in \mathrm{S}_{M}(m, \delta) \cap \operatorname{int}\left(\mathrm{B}_{M}(m, \delta)\right)$. Take $\varepsilon>0$ such that $\mathrm{U}_{M}(n, \varepsilon) \subseteq \mathrm{B}_{M}(m, \delta)$. Since $M$ satisfies the open property, there exists $s \in R$ with $|s|>1$ such that $s(n-m) \in \mathrm{U}_{M}(n-m, \varepsilon)$. Notice that

$$
|s-1| \delta=|s-1|\|n-m\|=\|s(n-m)-(n-m)\|<\varepsilon .
$$

Next,

$$
\|(m+s(n-m))-n\|=\|(m-n)+s(n-m)\|=|s-1|\|n-m\|=|s-1| \delta<\varepsilon .
$$

Therefore, $m+s(n-m) \in \mathrm{U}_{M}(n, \varepsilon) \subseteq \mathrm{B}_{M}(m, \delta)$. However,

$$
\|(m+s(n-m))-m\|=|s|\|n-m\|>\delta .
$$

This is a contradiction.

We present now a sufficient condition for a seminormed module to satisfy the open property.

Theorem 5. Let $R$ be a seminormed ring and $M$ a seminormed $R$-module. If $R$ satisfies the (strong) open property, then $M$ satisfies the (strong) open property.

Proof. Fix an open subset $U$ of $M$ and $m \in U$. Take $\varepsilon>0$ such that $\mathrm{U}_{M}(m, \varepsilon) \subseteq U$. Choose $\delta>0$ such that $\delta\|m\|<\varepsilon$. Then

$$
\mathrm{U}_{R}(1, \delta) m \subseteq \mathrm{U}_{M}(m, \delta\|m\|) \subseteq \mathrm{U}_{M}(m, \varepsilon) \subseteq U .
$$

By hypothesis, there exists $s \in R(s \in \mathcal{U}(R))$ with $\|s\|>1$ such that $s 1 \in \mathrm{U}_{R}(1, \delta)$. Then $s m \in U$.

According to Theorem 5, in order to guarantee that a seminormed module has the open property, it only suffices to find sufficient conditions for seminormed or absolutely semivalued rings to have the open property.

Corollary 2. The following are equivalent for an absolutely semivalued ring $R$ :

1. $\quad$ int $\left(\mathrm{B}_{R}(r, \delta)\right)=\mathrm{U}_{R}(r, \delta)$ for all $r \in R$ and all $\delta>0$.

2. $\quad \operatorname{int}\left(\mathrm{B}_{R}\right)=\mathrm{U}_{R}$.

3. $1 \notin \operatorname{int}\left(B_{R}\right)$.

4. $R$ satisfies the open property.

In this situation, $\mathrm{U}_{R}(r, \delta)$ is regular open for all $r \in R$ and all $\delta>0$.

Proof. It is trivial that $(1) \Rightarrow(2)$. Next, $(2) \Rightarrow(3)$ holds since $|1|=1$ (recall that we are assuming throughout the whole manuscript that all absolute semivalues are nonzero). Let us prove now that $(3) \Rightarrow(4)$. Fix an open subset $U$ of $R$ and $r \in U$. Take $\varepsilon>0$ such that $U_{R}(r, \varepsilon) \subseteq U$. If $|r|=0$, then $\mathrm{U}_{R}(1, \delta) r \subseteq \mathrm{U}_{R}(r, \varepsilon) \subseteq U$. If $|r|>0$, then we can choose $\delta>0$ such that $0<\delta|r|<\varepsilon$. Then $\mathrm{U}_{R}(1, \delta) r \subseteq \mathrm{U}_{R}(r, \delta|r|) \subseteq \mathrm{U}_{R}(r, \varepsilon) \subseteq U$. By hypothesis, we can find $s \in \mathrm{U}_{R}(1, \delta) \cap\left(R \backslash \mathrm{B}_{R}\right)$. Then $|s|>1$ and $s r \in U$. Finally, (4) $\Rightarrow(1)$ is a direct consequence of Theorem 4.

Scholium 6. Let $R$ be an absolutely semivalued ring such that $\mathcal{U}(R)$ is open. The following conditions are equivalent:

1. $\quad$ int $\left(\mathrm{B}_{R}(r, \delta)\right)=\mathrm{U}_{R}(r, \delta)$ for all $r \in R$ and all $\delta>0$.

2. $\quad \operatorname{int}\left(\mathrm{B}_{R}\right)=\mathrm{U}_{R}$.

3. $1 \notin \operatorname{int}\left(B_{R}\right)$. 
4. $\quad R$ satisfies the strong open property.

5. $R$ satisfies the open property.

Proof. In view of Corollary 2, we only need to show that $(3) \Rightarrow(4)$. It is essentially the same proof as in Corollary 2. Fix an open subset $U$ of $R$ and $r \in U$. Take $\varepsilon>0$ such that $U_{R}(r, \varepsilon) \subseteq U$. If $|r|=0$, then we choose $\delta>0$ such that $\mathrm{U}_{R}(1, \delta) \subseteq \mathcal{U}(R)$. Please note that $\mathrm{U}_{R}(1, \delta) r \subseteq \mathrm{U}_{R}(r, \varepsilon) \subseteq \mathcal{U}$. If $|r|>0$, then we can choose $\delta>0$ such that $\mathrm{U}_{R}(1, \delta) \subseteq \mathcal{U}(R)$ and $0<\delta|r|<\varepsilon$. Then $\mathrm{U}_{R}(1, \delta) r \subseteq \mathrm{U}_{R}(r, \delta|r|) \subseteq$ $\mathrm{U}_{R}(r, \varepsilon) \subseteq U$. By hypothesis, we can find $s \in \mathrm{U}_{R}(1, \delta) \cap\left(R \backslash \mathrm{B}_{R}\right)$. Then $s$ is invertible, $|s|>1$ and $s r \in U$.

In ([17] (Definition 3.12)), the concept of $w$-extreme point for rings was introduced: A point $e \in C$ is said to be a $w$-extreme point of a subset $C \subseteq R$ if the equality $2 e=a+b$, with $a, b \in C$, forces that $a=b$. By $\operatorname{ext}_{w}(C)$ we mean to denote the subset of $w$-extreme points of $C$. In ([16] (Theorem 4.1 and Theorem 4.3(1))), it was proved that $\operatorname{ext}_{w}(A) \cap \operatorname{int}(A)=\varnothing$ for every subset $A$ of a nondiscrete, integral domain, topological $\operatorname{ring} R$ with $\operatorname{char}(R) \neq 2$.

As a direct consequence of ([16] (Theorem 4.1 and Theorem 4.3(1))) together with Corollary 2 and Scholium 6, we immediately obtain the following result whose proof we omit for obvious reasons.

Theorem 7. Let $R$ be an absolutely semivalued ring. If $R$ is not discrete, $\operatorname{char}(R) \neq 2, R$ is an integral domain and $1 \in \operatorname{ext}_{w}\left(B_{R}\right)$, then $1 \notin \operatorname{int}\left(B_{R}\right)$ and hence $R$ satisfies the open property. If, in addition, $\mathcal{U}(R)$ is open, then $R$ satisfies the strong open property.

Observe that ([16] (Theorem 4.2)) is a direct consequence of Theorem 7 together with Theorem 4.

\section{Topological Implications of the Open Property}

This section deals with topological implications of the open property. In ([23] (Theorem 2)), it was proved that the intersection of all 0-neighborhoods in a topological module $M, \cap \mathcal{N}_{0}(M)$, is a closed submodule. If $M$ is seminormed, then $\bigcap \mathcal{N}_{0}(M)=\{m \in M:\|m\|=0\}$.

Lemma 1. Let $R$ be a seminormed ring and $M$ a seminormed $R$-module. If $p \in M$ and $\|p\|=0$, then $\|p+q\|=\|q\|$ for all $q \in M$.

Proof. It is sufficient to notice that $\|q\|=\|q\|-\|-p\| \leq\|q+p\| \leq\|q\|+\|p\|=\|q\|$.

Proposition 1. Let $R$ be an absolutely semivalued ring satisfying the open property. Let $M$ be a seminormed $R$-module. If $m \in M$ is such that $\|m+n\|=\|m\|$ for all $n$ in a closed ball $\mathrm{B}_{M}(0, t)$ with $t>0$, then $\|m\|=0$ and $\|n\|=0$ for all $n \in \mathrm{B}_{M}(0, t)$. As a consequence, $\cap \mathcal{N}_{0}(M)=\mathrm{B}_{M}(0, t)$, i.e., if $p \in M$ and $\|p\| \leq t$, then $\|p\|=0$.

Proof. Suppose on the contrary that $\|m\|>0$. Since $R$ satisfies the open property, there exists $s \in R$ with $|s|>1$ such that $s \in \mathrm{U}_{R}\left(1, \frac{t}{\|m\|}\right)$. Then $(s-1) m \in \mathrm{B}_{M}(0, t)$ and $\|m+(s-1) m\|=$ $\|m\|$. However,

$$
\|m+(s-1) m\|=|s|\|m\|>\|m\| .
$$

This is a contradiction. Therefore, $\|m\|=0$. By applying Lemma 1 , we have that $\|m+q\|=\|q\|$ for all $q \in M$. In particular, if $n \in \mathrm{B}_{M}(0, t)$, then $\|m+n\|=\|n\|$. However, by hypothesis, $\|m+n\|=\|m\|=0$, which implies that $\|n\|=0$. Then $\mathrm{B}_{M}(0, t) \subseteq \bigcap \mathcal{N}_{0}(M)$. Since $\mathrm{B}_{M}(0, t) \in \mathcal{N}_{0}(M)$, we conclude that $\mathrm{B}_{M}(0, t) \supseteq \bigcap \mathcal{N}_{0}(M)$. Then we obtain the equality $\mathrm{B}_{M}(0, t)=\bigcap \mathcal{N}_{0}(M)$.

Corollary 3. Let $R$ be an absolutely semivalued ring satisfying the open property. Let $M$ be a seminormed $R$-module. If $m \in M$ is such that $\|m+n\|=\|m\|$ for all $n$ in a closed ball $\mathrm{B}_{M}(p, t)$ with $p \in M$ and $t>0$, then $\|m\|=0$ and $\|n\|=0$ for all $n \in \mathrm{B}_{M}(p, t)$. As a consequence, $\cap \mathcal{N}_{0}(M)=\mathrm{B}_{M}(0, t)$. 
Proof. If $l \in \mathrm{B}_{M}(0, t)$, then $\|(m+p)+l\|=\|m+(p+l)\|=\|m\|=\|m+p\|$. According to Proposition $1,\|m+p\|=0$ and $\|l\|=0$ for all $l \in \mathrm{B}_{M}(0, t)$. This shows that $\cap \mathcal{N}_{0}(M)=\mathrm{B}_{M}(0, t)$. Notice that $\|m\|=\|m+p\|=0$. According to Lemma 1 and by using our hypothesis, $\|n\|=\|m+n\|=\|m\|=0$ for all $n \in \mathrm{B}_{M}(p, t)$.

Recall that a topological ring $R$ is called practical [23] if the invertibles approach 0, i.e., $0 \in$ $\operatorname{cl}(\mathcal{U}(R))$.

Theorem 8. Let $R$ be a practical topological ring and $M$ a topological $R$-module. Every proper submodule $N$ of $M$ has empty interior.

Proof. Suppose on the contrary that $N$ has nonempty interior. Then there exists an open set $V \subseteq N$. If we take any $p \in V$, then $V-p \subseteq N$ and $V-p$ is an open neighborhood of 0 . So, let us denote $U:=V-p$. Fix any arbitrary $m \in M$. There is a 0-neighborhood $W$ in $R$ with $W m \subseteq U$. Next, $R$ is practical, so there is an invertible $u \in \mathcal{U}(R)$ verifying that $u \in W$. Then $m=u^{-1}(u m) \in u^{-1} W m \subseteq u^{-1} N=N$. The arbitrariness of $m \in M$ implies that $M \subseteq N$ and thus $N$ is not proper.

As an immediate consequence of Theorem 8 and Corollary 3, we have the next result.

Corollary 4. Let $R$ be a practical absolutely semivalued ring satisfying the open property. Let $M$ be a seminormed $R$-module. For every $m, p \in M$ and every $t>0$ there exists $n \in \mathrm{B}_{M}(p, t)$ such that $\|m+n\| \neq\|m\|$.

Proof. We are assuming that all seminorms are not zero, therefore $\cap \mathcal{N}_{0}(M)$ is a proper submodule of $M$ in virtue of ([23] (Theorem 2)). Then Theorem 8 implies that $\cap \mathcal{N}_{0}(M)$ has empty interior. Finally, Corollary 3 forces that for every $m, p \in M$ and every $t>0$ there exists $n \in \mathrm{B}_{M}(p, t)$ such that $\|m+n\| \neq\|m\|$.

\section{More Examples of Unit Neighborhoods of Zero}

By means of the open property, we will be able to provide more new examples of unit neighborhoods of zero ([17] (Definition 2.2)). A regular open 0-neighborhood in a topological ring is called an open-unit 0-neighborhood if it is additively symmetric, multiplicatively idempotent and 1 belongs to its closure. A closed-unit 0-neighborhood is a regular closed 0-neighborhood whose interior is an open-unit 0-neighborhood. The closure of each open-unit 0-neighborhood is a closed-unit 0-neighborhood. The first examples of closed- and open-unit 0-neighborhoods were given in ([17] (Theorem 3.7)) and in [29], in the context of $\mathbb{R}$ and $\mathbb{C}$, respectively. Later on, more examples were given in ([16] (Proposition 3.1)), where it was shown that if $A$ is an absolutely semivalued unital real algebra, then $B_{A}$ is a closed-unit 0-neighborhood. This result was generalized to normed rings in ([23] (Theorem 8)) with few extra hypotheses.

Theorem 9. Let $A$ be a unital seminormed algebra over an absolutely semivalued ring $R$. If, in addition, $A$ is an inversion-topological ring, satisfies the open property (with respect to $R$ ) and $1 \in \operatorname{cl}\left(\mathrm{U}_{R} \cap \mathcal{U}(R)\right.$ ), then $\mathrm{U}_{A}=\operatorname{int}\left(\mathrm{B}_{A}\right)$ and thus it is an open-unit 0-neighborhood, and $\mathrm{B}_{A}=\operatorname{cl}\left(\mathrm{U}_{A}\right)$ and thus it is a closed-unit 0-neighborhood.

Proof. In the first place, in virtue of Theorems 2 and $4, \mathrm{U}_{A}=\operatorname{int}\left(\mathrm{B}_{A}\right)$ and $\mathrm{B}_{A}=\mathrm{cl}\left(\mathrm{U}_{A}\right)$, so $\mathrm{U}_{A}$ is regular open and $\mathrm{B}_{A}$ is regular closed. Thus, it only suffices to show that $\mathrm{U}_{A}$ is an open-unit neighborhood of 0 . Since $\mathrm{U}_{A}$ is regular open, it only remains to show that $\mathrm{U}_{A}$ is additively symmetric, multiplicatively idempotent and $1 \in \operatorname{cl}\left(\mathrm{U}_{A}\right)$. It is clear that $\mathrm{U}_{A}$ is additively symmetric and $1 \in \mathrm{B} A=\mathrm{cl}\left(\mathrm{U}_{A}\right)$ because $A$ is unital so $\|1\|=1$. It is also trivial that $\mathrm{U}_{A} \mathrm{U}_{A} \subseteq \mathrm{U}_{A}$. So, it only remains to show that $\mathrm{U}_{A} \subseteq \mathrm{U}_{A} \mathrm{U}_{A}$. For this, we will rely on ([18] (Lemma 2.4)). By hypothesis, $1 \in \mathrm{cl}\left(\mathrm{U}_{R} \cap \mathcal{U}(R)\right)$, thus we can find a 
sequence $\left(u_{n}\right)_{n \in \mathbb{N}} \subseteq \mathrm{U}_{R} \cap \mathcal{U}(R)$ converging to 1 in $R$. Then the sequence $\left(u_{n} 1\right)_{n \in \mathbb{N}}$ is contained in $\mathrm{U}_{A} \cap \mathcal{U}(A)$ and converges to 1 in $A$. Therefore, $1 \in \operatorname{cl}\left(U_{A} \cap \mathcal{U}(A)\right)$. Finally, ([18] (Lemma 2.4)) assures that $\mathrm{U}_{A} \subseteq \mathrm{U}_{A} \mathrm{U}_{A}$ because multiplicative inversion on $A$ is continuous by hypothesis.

Theorem 9 generalizes ([16] (Proposition 3.1)).

Corollary 5. If $A$ is an absolutely semivalued unital real algebra, then $\mathrm{U}_{A}=\operatorname{int}\left(\mathrm{B}_{A}\right)$ and thus it is an open-unit 0-neighborhood, and $\mathrm{B}_{A}=\mathrm{cl}\left(\mathrm{U}_{A}\right)$ and thus it is a closed-unit 0-neighborhood.

Proof. Since $A$ is absolutely semivalued, we have that $A$ is unital and inversion on $A$ is continuous. According to Corollary 2, $\mathbb{R}$ clearly satisfies the open property and so does $A$ with respect to $\mathbb{R}$ by Theorem 5 . Next, it is obvious that $1 \in \operatorname{cl}\left(\cup_{\mathbb{R}} \cap \mathcal{U}(\mathbb{R})\right)$. Therefore, it only suffices to call on Theorem 9.

We conclude this section with more corollaries of Theorem 9.

Corollary 6. If $A$ is a unital real Banach algebra, then $\mathrm{U}_{A}=\operatorname{int}\left(\mathrm{B}_{A}\right)$ and thus it is an open-unit 0-neighborhood, and $\mathrm{B}_{A}=\mathrm{cl}\left(\mathrm{U}_{A}\right)$ and thus it is a closed-unit 0-neighborhood.

Proof. Since $A$ is a unital real Banach algebra, we have that inversion on $A$ is continuous because of the completeness of $A$ (see, for instance, ([25] (Theorem 4.2))). According to Corollary 2, $\mathbb{R}$ clearly satisfies the open property and so does $A$ with respect to $\mathbb{R}$ by Theorem 5 . Next, it is obvious that $1 \in \operatorname{cl}\left(\cup_{\mathbb{R}} \cap \mathcal{U}(\mathbb{R})\right)$. Thus, we only need to apply Theorem 9 .

Corollary 7. Let $R$ be an absolutely semivalued ring. If $R$ satisfies the open property and $1 \in \operatorname{cl}\left(\mathrm{U}_{R} \cap \mathcal{U}(R)\right)$, then $\mathrm{U}_{R}=\operatorname{int}\left(\mathrm{B}_{R}\right)$ and thus it is an open-unit 0-neighborhood, and $\mathrm{B}_{R}=\mathrm{cl}\left(\mathrm{U}_{R}\right)$ and thus it is a closed-unit 0-neighborhood.

Proof. Here we take $A:=R$ and then, since $A$ is absolutely semivalued, $A$ is unital and inversion on $A$ is continuous. Next, $R$ satisfies the open property and $1 \in \operatorname{cl}\left(\mathrm{U}_{R} \cap \mathcal{U}(R)\right)$ by hypothesis. In view of Theorem 9 , we obtain the desired result.

We refer the reader to ([23] (Theorem 8)) for sufficient conditions on complete normed rings for their unit ball to be a closed-unit neighborhood of 0 .

\section{The Open Property in Topological Modules}

In this section, we will extend the open property to topological modules over topological rings.

Definition 2. Let $R$ be a topological ring and $B$ a proper closed-unit 0-neighborhood of $R$. We say that a topological $R$-module $M$ satisfies the (strong) open property if for every open subset $U$ of $M$ and for every $m \in U$ there is an (invertible) element $s \in R \backslash B$ with $s m \in U$.

A topological ring satisfies the (strong) open property it does as a left module over itself. The next result extends Theorem 5 to topological modules.

Theorem 10. Let $R$ be a topological ring, $B$ a proper closed-unit 0-neighborhood of $R$ and $M$ a topological $R$-module. If $R$ satisfies the (strong) open property, then $M$ satisfies the (strong) open property.

Proof. Fix an open subset $U$ of $M$ and $m \in U$. Take $V$ an open neighborhood of 1 in $R$ with $V m \subseteq U$. There is $s \in R(s \in \mathcal{U}(R))$ with $s \notin B$ and $s 1 \in V$. Then $s m \in U$.

According to Theorem 10, in order to guarantee that a topological module has the open property, it only suffices to find sufficient conditions for topological rings to have the open property. 
Proposition 2. Let $R$ be a topological ring and $B$ a proper closed-unit 0-neighborhood of $R$. Then $R$ satisfies the open property if and only if $1 \notin \operatorname{int}(B)$.

Proof. Suppose first that $R$ satisfies the open property. If $1 \in \operatorname{int}(B)$, then there exists $s \in R \backslash B$ such that $s=s 1 \in \operatorname{int}(B) \subseteq B$. This is a contradiction. Conversely, suppose that $1 \notin \operatorname{int}(B)$. Fix a nonempty open subset $U$ of $R$ and $r \in U$. There is an additively symmetric 0 -neighborhood $V$ of $R$ satisfying that if $v \in V$, then $(1 \pm v) r \in U$. Since $1 \in \operatorname{bd}(B)$, we have that $(1+V) \cap(R \backslash B) \neq \varnothing$. Therefore, we can fix $v_{0} \in V$ such that $s:=1+v_{0} \notin B$.

Proposition 3. Let $R$ be a topological ring and $B$ a proper closed-unit 0-neighborhood of $R$. Suppose that $\mathcal{U}(R)$ is open. The following conditions are equivalent:

1. $R$ satisfies the open property.

2. $1 \notin \operatorname{int}(B)$.

3. $R$ satisfies the strong open property.

Proof. In view of Proposition 2, it only remains to prove (2) $\Rightarrow(3)$. Fix a nonempty open subset $U$ of $R$ and $r \in U$. There is an additively symmetric 0 -neighborhood $V$ of $R$ verifying that if $v \in V$, then $(1 \pm v) r \in U$. Since $1 \in \operatorname{bd}(B)$, we have that $(1+V) \cap(R \backslash B) \neq \varnothing$. Therefore, we can fix $v_{0} \in V$ such that $s:=1+v_{0} \notin B$. Finally, since $\mathcal{U}(R)$ is open, then $V$ can be chosen to verify also that $1+V \subseteq \mathcal{U}(R)$.

As a direct consequence of ([16] (Theorem 4.1 and Theorem 4.3(1))) together with Propositions 2 and 3 , we immediately obtain the following result whose proof we omit for obvious reasons.

Theorem 11. Let $R$ be a topological ring and $B$ a proper closed-unit 0-neighborhood of $R$. If $R$ is not discrete, $\operatorname{char}(R) \neq 2, R$ is an integral domain and $1 \in \operatorname{ext}_{w}(B)$, then $1 \notin \operatorname{int}(B)$ and hence $R$ satisfies the open property. If, in addition, $\mathcal{U}(R)$ is open, then $R$ satisfies the strong open property.

This final example displays a topological module that fails the open property.

Example 3. Consider an integral domain $R$ and $M$ a torsionfree $R$-module. Assume that both $R$ and $M$ are endowed with the discrete topology. Then $M$ fails the open property with respect to any proper closed-unit neighborhood $B$ of 0 in $R$ (an example of a proper closed-unit neighborhood of 0 in $R$ is $\{-1,0,1\}$ ). Indeed, take any $m \in M \backslash\{0\}$. Please note that $\{m\}$ is open in $M$. If $s \in R \backslash B$, then sm $\notin\{m\}$ because $M$ is torsionfree and $R$ is an integral domain.

\section{The $\ell_{\infty}$-Space}

This section is devoted to show examples of topological rings satisfying the hypotheses of Propositions 2 and 3 . We will rely on $\ell_{\infty}$-spaces to construct these examples.

If $\Gamma$ is a nonempty set, $R$ is a topological ring and $X$ is a topological $R$-module, then

$$
\ell_{\infty}(\Gamma, X):=\left\{f \in X^{\Gamma}: f(\Gamma) \text { is bounded in } X\right\} .
$$

Recall that a subset $A \subseteq X$ is said to be bounded if for each 0-neighborhood $U$ in $X$ there is an invertible $u \in \mathcal{U}(R)$ such that $A \subseteq u U$. In case $R$ and $X$ are seminormed, then the boundedness concept usually considered in $X$ is the one given by the seminorm, i.e., a subset is bounded if it is contained in a ball centered at 0 . In this situation, $\ell_{\infty}(\Gamma, X)$ acquires structure of seminormed $R$-module, where

$$
\|f\|_{\infty}:=\sup \{\|f(\tau)\|: \tau \in \Gamma\}
$$


If $A$ is seminormed $R$-algebra, then $\ell_{\infty}(\Gamma, A)$ acquires structure of seminormed $R$-algebra.

Example 4. Let $\Gamma$ be a nonempty set and consider the $\mathbb{K}$-Banach algebra $(\mathbb{K}:=\mathbb{R}, \mathbb{C})$

$$
\ell_{\infty}(\Gamma):=\left\{f \in \mathbb{K}^{\Gamma}: f(\Gamma) \text { is bounded in } \mathbb{K}\right\}
$$

with the sup-norm

$$
\|f\|_{\infty}:=\sup \{|f(\tau)|: \tau \in \Gamma\} .
$$

Observe that $\ell_{\infty}(\Gamma)$ is not absolutely semivalued, thus Theorem 7 does not apply. Also, $\ell_{\infty}(\Gamma)$ is not discrete and char $\left(\ell_{\infty}(\Gamma)\right) \neq 2$, but $\ell_{\infty}(\Gamma)$ is not an integral domain. Thus, Theorem 11 does not apply either. On the other hand, the unit ball $\mathrm{B}_{\ell_{\infty}(\Gamma)}$ is clearly a closed-unit neighborhood of 0 (see Corollary 6 ) such that 1 is an extreme point of $\mathrm{B}_{\ell_{\infty}(\Gamma)}$. Indeed, if $2=f+g$ with $f, g \in \mathrm{B}_{\ell_{\infty}(\Gamma)}$, then $2=f(\tau)+g(\tau)$ for all $\tau \in \Gamma$. Since $|f(\tau)| \leq 1$ and $|g(\tau)| \leq 1$, the only possibility is that $f(\tau)=g(\tau)=1$ for all $\tau \in \Gamma$. Therefore, $f=g=1$. In particular, $1 \notin$ int $\left(\mathrm{B}_{\ell_{\infty}(\Gamma)}\right)=\mathrm{U}_{\ell_{\infty}(\Gamma)}$. Bearing in mind Proposition 2, all of these imply that $\ell_{\infty}(\Gamma)$ satisfies the open property (with respect to itself). As a consequence, Theorem 10 assures that any topological module over $\ell_{\infty}(\Gamma)$ satisfies the open property. Also,

$$
\mathcal{U}\left(\ell_{\infty}(\Gamma)\right)=\left\{f \in \ell_{\infty}(\Gamma): \inf _{\tau \in \Gamma}|f(\tau)|>0\right\}
$$

is clearly open in $\ell_{\infty}(\Gamma)$. Therefore, by Proposition $2, \ell_{\infty}(\Gamma)$ satisfies the strong open property (with respect to itself), and hence any topological module over $\ell_{\infty}(\Gamma)$ satisfies the strong open property (Theorem 10).

The following proposition shows that norm-boundedness and boundedness coincide when the underlying ring is practical.

Proposition 4. Let $R$ be an absolutely semivalued ring and $M$ a seminormed $R$-module. If $A \subseteq M$ is bounded, then it is seminorm-bounded. Conversely, if $R$ is practical and $A \subseteq M$ is seminorm-bounded, then it is bounded.

Proof. Assume first that $A \subseteq M$ is bounded. There is $u \in \mathcal{U}(R)$ with $A \subseteq u \mathrm{~B}_{M}=\mathrm{B}_{M}(0,|u|)$, which means that $A$ is seminorm-bounded. Conversely, assume that $R$ is practical and $A \subseteq M$ is seminorm-bounded. Fix any arbitrary 0-neighborhood $V$ in $M$. Take $t>0$ with $\mathrm{B}_{M}(0, t) \subseteq V$. Since $A$ is seminorm-bounded, there exists $s>0$ with $A \subseteq \mathrm{B}_{M}(0, s)$. Since $R$ is practical, we can find an invertible $v \in R$ with $|u| \leq \frac{t}{s}$. Then

$$
A \subseteq \mathrm{B}_{M}(0, s) \subseteq \mathrm{B}_{M}\left(0,|u|^{-1} t\right)=u^{-1} \mathrm{~B}_{M}(0, t) \subseteq u^{-1} V
$$

We will finalize this section by providing sufficient conditions on a topological module $M$ for $\ell_{\infty}(\Gamma, M)$ to have additive group structure.

Lemma 2. Let $R$ be a practical topological ring and $M$ a topological $R$-module. If $A, B \subseteq M$ are bounded, then $A+B$ is also bounded.

Proof. Fix an arbitrary 0-neighborhood $U$ in $M$. Take $V$ a 0-neighborhood in $M$ with $V+V \subseteq U$. There are neighborhoods $W_{1}, W_{2}$ of 0 in $R$ and $M$, respectively, such that $W_{1} W_{2} \subseteq V$. Since $A, B$ are bounded, we can find invertibles $r, s \in R$ with $A \subseteq r W_{2}$ and $B \subseteq s W_{2}$. Next, there exist neighborhoods $E, F$ of 0 in $R$ such that $E r \subseteq W_{1}$ and $F s \subseteq W_{1}$. Since $R$ is practical, there exists $t \in \mathcal{U}(R)$ with $t \in E \cap F$. Then $t r \in E r \subseteq W_{1}$ and $t s \in F s \subseteq W_{1}$. This means that

$$
t\left(r W_{2}+s W_{2}\right)=(t r) W_{2}+(t s) W_{2} \subseteq W_{1} W_{2}+W_{1} W_{2} \subseteq V+V \subseteq U,
$$


in other words,

$$
A+B \subseteq r W_{2}+s W_{2} \subseteq t^{-1} U .
$$

This proves that $A+B$ is bounded.

A direct consequence of Lemma 2 is the fact that $\ell_{\infty}(\Gamma, M)$ is a commutative additive group as long as $R$ is practical.

Theorem 12. Let $R$ be a practical topological ring and $M$ a topological $R$-module. If $\Gamma$ is a nonempty set, then $\ell_{\infty}(\Gamma, M)$ acquires structure of commutative additive group with the regular addition of M-valued functions.

Proof. If $f, g \in \ell_{\infty}(\Gamma, M)$, then $(f+g)(\Gamma) \subseteq f(\Gamma)+g(\Gamma)$ and $f(\Gamma)+g(\Gamma)$ is bounded in virtue of Lemma 2.

\section{Regularity in Topological Modules}

Recall that in Theorems 2 and 4, sufficient conditions where provided to assure that open balls and closed balls are regular in seminormed modules over absolutely semivalued rings. We will study in this section how to transport those results to topological modules. We will begin by versioning Theorem 2 for topological modules.

Definition 3. Let $R$ be a topological ring, $B$ a closed-unit 0-neighborhood in $R$ and $M$ a topological $R$-module. $A$ subset $C \subseteq M$ is called internally $B$-balanced if $\operatorname{int}(B) C \subseteq \operatorname{int}(C)$.

Observe that internally $B$-balanced sets are necessarily zero-neighborhoods.

Example 5. Let $R$ be a seminormed ring such that $B:=B_{R}$ is a closed-unit 0-neighborhood with int $\left(B_{R}\right)=U_{R}$. If $M$ is a seminormed $R$-module, then every closed ball of $M$ centered at 0 is internally B-balanced. Indeed, if $t>0$, then

$$
\operatorname{int}\left(\mathrm{B}_{R}\right) \mathrm{B}_{M}(0, t)=\mathrm{U}_{R} \mathrm{~B}_{M}(0, t) \subseteq \mathrm{U}_{M}(0, t) \subseteq \operatorname{int}\left(\mathrm{B}_{M}(0, t)\right) .
$$

Theorem 13. Let $R$ be a topological ring, $B$ a closed-unit 0-neighborhood in $R$ and $M$ a topological $R$-module. If $F$ is an internally B-balanced, closed 0-neighborhood in $M$, then $F$ is regular closed.

Proof. We know that $\mathrm{cl}(\operatorname{int}(F)) \subseteq F$. Let $x \in F$ and take $V$ any 0 -neighborhood in $M$. There is a 0-neighborhood $U$ in $R$ such that $U x \subseteq V$. Since $1 \in \operatorname{cl}(\operatorname{int}(B))$, there is $b \in \operatorname{int}(B)$ with $b \in 1+U$, i.e., $b=1+u$ for some $u \in U$. Then $b x \in \operatorname{int}(B) F \subseteq \operatorname{int}(F)$ and $b x=(1+u) x=x+u x \in x+U x \subseteq x+V$.

The upcoming results are devoted to somehow extend Theorem 4 to the category of topological modules.

Definition 4. Let $R$ be a topological ring and $M$ a topological $R$-module. A functional $m^{*} \in M^{*}$ is said to be open if it is an open map.

The following positive lemma shows examples of open functionals.

Lemma 3. Let $R$ be a topological ring and $M$ a topological $R$-module. If $m^{*} \in M^{*}$ satisfies that $m^{*}(M) \cap \mathcal{U}(R) \neq \varnothing$, then $m^{*}$ is open.

Proof. It only suffices to show that if $W$ is a 0-neighborhood in $M$, then $m^{*}(W)$ is a 0 -neighborhood in $R$. Indeed, fix $m \in M$ with $m^{*}(m) \in \mathcal{U}(R)$. In view of Theorem 1(5), there is a 0-neighborhood $U \subseteq R$ 
verifying that $U m \subseteq V$. Then $U m^{*}(m) \subseteq m^{*}(V)$. Since $m^{*}(m)$ is invertible, we have that $U m^{*}(m)$ is a neighborhood of 0 in $R$ and so is $m^{*}(V)$.

The following negative lemma, shows examples of nonopen functionals.

Lemma 4. Let $R$ be an integral domain Hausdorff topological ring for which there exists $s_{0} \in R \backslash\{0\}$ such that $\operatorname{int}\left(R s_{0}\right)=\varnothing$. Let $M$ be a strongly torsionfree topological $R$-module such that there exists $m_{0} \in M \backslash\{0\}$ and a continuous linear projection $P: M \rightarrow M$ with $P(M)=R m_{0}$. Consider the continuous $R$-linear map

$$
\begin{aligned}
Q: R m_{0} & \rightarrow R \\
r m_{0} & \mapsto r s_{0} .
\end{aligned}
$$

Then the continuous linear functional $m^{*}:=Q \circ P$ is not open.

Proof. Let us show first that $Q$ is well defined. Indeed, if $r m_{0}=s m_{0}$, then $(r-s) m_{0}=0$, so the constant net $r-s$ converges to a zero divisor because $m_{0} \neq 0$ and $M$ is strongly torsionfree. Since $R$ is an integral domain and Hausdorff, we conclude that $r-s=0$ and hence $r=s$. This shows that $Q$ is well defined. Please note that $Q$ is $R$-linear. The continuity of $Q$ follows from the fact that $M$ is strongly torsionfree and $R$ is Hausdorff. Indeed, let $\left(r_{i} m_{0}\right)_{i \in I}$ be a net convergent to some $r m_{0}$. Then $\left(\left(r_{i}-r\right) m_{0}\right)_{i \in I}$ is convergent to 0 . Since $m_{0} \neq 0$ and $M$ is strongly torsionfree, $\left(r_{i}-r\right)_{i \in I}$ converges to a zero divisor. However, $R$ is an integral domain, thus $\left(r_{i}-r\right)_{i \in I}$ converges to 0 and hence $\left(r_{i}\right)_{i \in I}$ converges to $r$, so $\left(r_{i} s_{0}\right)_{i \in I}$ converges to $r s_{0}$. This proves the continuity of $Q$. Since $P$ is linear and continuous by hypothesis, we have that $m^{*} \in M^{*}$. It only remains to show that $m^{*}$ is not open. Notice that $m^{*}(M)=Q(P(M))=Q\left(R m_{0}\right)=R s_{0}$, which means that $\operatorname{int}\left(m^{*}(M)\right)=\operatorname{int}\left(R s_{0}\right)=\varnothing$ and hence $m^{*}$ cannot be open.

Example 6. Given any absolutely valued ring $R$, an example of an $R$-module verifying the hypotheses of Lemma 4 is $M:=R \times R$ endowed with the product topology. Indeed, $M$ is strongly torsionfree because $R$ is absolutely valued and if we take $m_{0}:=(1,0)$, then

$$
\begin{aligned}
P: \quad R \times R & \rightarrow R \times R \\
(r, s) & \mapsto(r, 0)
\end{aligned}
$$

is a continuous linear projection such that $P(R \times R)=R \times\{0\}=R(1,0)$.

Example 7. A ring satisfying the hypotheses of Lemma 4 is, for instance, $R:=\mathbb{Q}[\pi]$ with the usual absolute value and with $s_{0}:=\pi$. Please note that $\pi$ is not invertible in $\mathbb{Q}[\pi]$ because $\pi$ is transcendental over $\mathbb{Q}$. Also, $\mathbb{Q}[\pi] \pi$ has empty interior in $\mathbb{Q}[\pi]$ since

$$
\mathbb{Q} \backslash\{0\} \subseteq \mathbb{Q}[\pi] \backslash \mathbb{Q}[\pi] \pi .
$$

Indeed, if there are $q_{0} \in \mathbb{Q} \backslash\{0\}$ and $q(x) \in \mathbb{Q}[x]$ such that $q_{0}=q(\pi) \pi$, then $q_{0}-q(\pi) \pi=0$, so if we consider the polynomial $a(x):=q_{0}-q(x) x$, then $a(x) \in \mathbb{Q}[x]$ and $a(\pi)=0$. Since $\pi$ is transcendental over $\mathbb{Q}$, it must occur that $a(x)=0$, i.e., $q_{0}=q(x) x$, which implies that $q_{0}=q(x)=0$. This contradicts the fact that $q_{0} \neq 0$. This shows that $\mathbb{Q} \backslash\{0\} \subseteq \mathbb{Q}[\pi] \backslash \mathbb{Q}[\pi] \pi$ and then $\mathbb{Q}[\pi] \backslash \mathbb{Q}[\pi] \pi$ is dense in $\mathbb{Q}[\pi]$ which means that $\mathbb{Q}[\pi] \pi$ has empty interior in $\mathbb{Q}[\pi]$.

Lemma 5. Let $R$ be a topological ring and $M$ a topological $R$-module. Take $m^{*} \in M^{*}$ open. If $A \subseteq R$, then $\left(m^{*}\right)^{-1}(\operatorname{int}(A))=\operatorname{int}\left(\left(m^{*}\right)^{-1}(A)\right)$ and $\mathrm{cl}\left(\left(m^{*}\right)^{-1}(A)\right)=\left(m^{*}\right)^{-1}(\mathrm{cl}(A))$.

Proof. The continuity of $m^{*}$ implies that $\left(m^{*}\right)^{-1}(\operatorname{int}(A))$ is open in $M$ and $\left(m^{*}\right)^{-1}(\operatorname{cl}(A))$ is closed in $M$. Therefore, $\left(m^{*}\right)^{-1}(\operatorname{int}(A)) \subseteq \operatorname{int}\left(\left(m^{*}\right)^{-1}(A)\right)$ and $\operatorname{cl}\left(\left(m^{*}\right)^{-1}(A)\right) \subseteq\left(m^{*}\right)^{-1}(\operatorname{cl}(A))$. Let us 
prove first that $\left(m^{*}\right)^{-1}(\operatorname{int}(A)) \supseteq \operatorname{int}\left(\left(m^{*}\right)^{-1}(A)\right)$. Take an element $m \in \operatorname{int}\left(\left(m^{*}\right)^{-1}(A)\right)$. There is a 0-neighborhood $V \subseteq M$ with $m+V \subseteq\left(m^{*}\right)^{-1}(A)$. Then $m^{*}(m)+m^{*}(V) \subseteq A$. By hypothesis, since $m^{*}$ is open, we conclude that $m^{*}(V)$ is a neighborhood of 0 in $R$. Then $m^{*}(m) \in \operatorname{int}(A)$, which implies that $m \in\left(m^{*}\right)^{-1}(\operatorname{int}(A))$. This shows that $\left(m^{*}\right)^{-1}(\operatorname{int}(A)) \supseteq \operatorname{int}\left(\left(m^{*}\right)^{-1}(A)\right)$ and hence the equality. Let us prove next that $\mathrm{cl}\left(\left(m^{*}\right)^{-1}(A)\right) \supseteq\left(m^{*}\right)^{-1}(\operatorname{cl}(A))$. Take an element $m \in\left(m^{*}\right)^{-1}(\mathrm{cl}(A))$. Fix any arbitrary neighborhood $V$ of 0 in $M$. By hypothesis, since $m^{*}$ is open, we conclude that $m^{*}(V)$ is a 0-neighborhood of $R$. Hence, $\left(m^{*}(m)+m^{*}(V)\right) \cap A \neq \varnothing$ because $m^{*}(m) \in \operatorname{cl}(A)$ by assumption. This means that $(m+V) \cap\left(m^{*}\right)^{-1}(A) \neq \varnothing$, i.e., $m \in \operatorname{cl}\left(\left(m^{*}\right)^{-1}(A)\right)$. This shows that $\mathrm{cl}\left(\left(m^{*}\right)^{-1}(A)\right) \supseteq\left(m^{*}\right)^{-1}(\mathrm{cl}(A))$ and hence the equality.

Theorem 14. Let $R$ be a topological ring and $M$ a topological $R$-module. Take $m^{*} \in M^{*}$ open. If $B \subseteq R$ is a closed-unit neighborhood of 0 , then $\operatorname{int}\left(\left(m^{*}\right)^{-1}(B)\right)=\left(m^{*}\right)^{-1}(\operatorname{int}(B))$ and $\operatorname{cl}\left(\left(m^{*}\right)^{-1}(\operatorname{int}(B))\right)=\left(m^{*}\right)^{-1}(B)$. In particular, $\left(m^{*}\right)^{-1}(\operatorname{int}(B))$ is regular open and $\left(m^{*}\right)^{-1}(B)$ is regular closed.

Proof. By applying Lemma 5 and taking into consideration that $B$ is regular closed,

$$
\operatorname{cl}\left(\left(m^{*}\right)^{-1}(\operatorname{int}(B))\right)=\left(m^{*}\right)^{-1}(\operatorname{cl}(\operatorname{int}(B)))=\left(m^{*}\right)^{-1}(B) .
$$

To see that $\left(m^{*}\right)^{-1}(\operatorname{int}(B))$ is regular open, it suffices to apply Lemma 5 because

$$
\operatorname{int}\left(\operatorname{cl}\left(\left(m^{*}\right)^{-1}(\operatorname{int}(B))\right)\right)=\operatorname{int}\left(\left(m^{*}\right)^{-1}(B)\right)=\left(m^{*}\right)^{-1}(\operatorname{int}(B)) .
$$

To see that $\left(m^{*}\right)^{-1}(B)$ is regular closed, it suffices to apply Lemma 5 because

$$
\operatorname{cl}\left(\operatorname{int}\left(\left(m^{*}\right)^{-1}(B)\right)\right)=\operatorname{cl}\left(\left(m^{*}\right)^{-1}(\operatorname{int}(B))\right)=\left(m^{*}\right)^{-1}(\operatorname{cl}(\operatorname{int}(B)))=\left(m^{*}\right)^{-1}(B) .
$$

\section{Balanced and Absorbing Subsets Which Are Not Zero-Neighborhoods}

This final section is aimed at generalizing ([14] (Theorem 3.2)) and ([15] (Theorem 1.1)) to the scope of topological modules. For this, we need first to introduce the concepts of balancedness and absorbance for topological modules.

The following definition can be found in ([16] (Definition 2.1)) and extends the geometrical concepts of balanced and absorbance from topological vector spaces to modules.

Definition 5. Let $R$ be a topological ring and $M$ an $R$-module. Consider a closed-unit zero-neighborhood $B \subseteq R$. A nonempty subset $A$ of $M$ is said to be

- B-balanced provided that $B A=A$;

- $\quad B$-absorbing provided that for all $m \in M$ there exists an invertible element $u \in \mathcal{U}(R)$ such that $B m \subseteq u A$.

Observe that not every topological module over a topological ring has a balanced and absorbing subset which is not a zero-neighborhood. This is an example where pathological phenomena occur in topological modules.

Example 8. Indeed, if $M$ is an $R$-module and we endow both $R$ and $M$ with the discrete topology, then every subset of $M$ is trivially open and thus every B-balanced and B-absorbing subset of $M$ is a neighborhood of 0 , where $B$ can be chosen, for instance, to be $\{-1,0,1\}$. 
The following lemma justifies why ([15] (Theorem 1.1)) works for real dimensions greater than or equal to 2 .

Lemma 6. If $R$ is a topological ring and $B \subseteq R$ is a closed-unit zero-neighborhood, then every B-absorbing subset $A \subseteq R$ is a neighborhood of 0 .

Proof. For $1 \in R$ there exists an invertible element $u \in \mathcal{U}(R)$ such that $B=B 1 \subseteq u A$. Then $u^{-1} B \subseteq A$. Since $u^{-1} B$ is a neighborhood of 0 , then so is $A$.

To construct a balanced and absorbing subset which is not a zero-neighborhood, we will need to rely on a special class of closed-unit zero-neighborhoods. The following definition is a compilation of ([18] (Definition 2.16)) and ([16] (Definition 2.3)).

Definition 6. Let $R$ be a topological ring and $B \subseteq R$ a closed-unit 0-neighborhood. Then $B$ is called left-feasible provided that $\{u B: u \in \mathcal{U}(R)\}$ is a basis of neighborhoods of 0 .

Proposition 5. Let $R$ be a topological ring and $M$ a topological $R$-module. If there exists a left-feasible closed-unit 0-neighborhood $B \subseteq R$, then every neighborhood $U$ of 0 in $M$ is B-absorbing.

Proof. Fix an arbitrary $m \in M$. There exists a 0 -neighborhood $V \subseteq R$ such that $V m \subseteq U$. Since $B$ is left-feasible, we can find an invertible $u \in \mathcal{U}(R)$ with $u B \subseteq V$. Then $u B m \subseteq V m \subseteq U$, so $B m \subseteq u^{-1} U$. This shows that $U$ is $B$-absorbing.

In a module $M$ over a ring $R$, two elements $m, n \in M$ are said to be equivalent when there exists an invertible $u \in \mathcal{U}(R)$ such that $m=u n$. This is clearly an equivalence relation on $M$, which we will denote by $\mathcal{R}$. Observe that the equivalence classes are given by $[m]_{\mathcal{R}}=\mathcal{U}(R) m$, for all $m \in M$. A subset $A \subseteq M$ is said to be $\mathcal{R}$-free provided that for every $a, b \in A$ with $a \neq b$, we have that $[a]_{\mathcal{R}} \neq[b]_{\mathcal{R}}$.

Lemma 7. Let $R$ be a ring and $M$ an $R$-module. If $A \subseteq M$ is not empty, then there exists a maximal $\mathcal{R}$-free subset $D \subseteq A$.

Proof. Define $\mathcal{L}:=\{C \subseteq A: C$ is $\mathcal{R}$-free $\}$ partially ordered by the inclusion. Notice that $\mathcal{L} \neq \varnothing$ because if $a \in A$, then $C:=\{a\} \in \mathcal{L}$. Next, $\mathcal{L}$ is an inductive set in the sense that every chain of $\mathcal{L}$ has an upper bound which consists of the union of the elements of the chain. Finally, Zorn's Lemma assures the existence of a maximal element in $\mathcal{L}$, i.e., a maximal $\mathcal{R}$-free subset $D \subseteq A$.

We are now in the right position to begin the construction of balanced and absorbing subsets which are not zero-neighborhoods.

Lemma 8. Let $R$ be a topological ring and $M$ an $R$-module. Consider a closed-unit zero-neighborhood $B \subseteq R$. If $P \subseteq M$, then $A:=\bigcup_{p \in P} B p$ is $B$-balanced. If, in addition, $B$ is left-feasible and $P$ is a maximal $\mathcal{R}$-free subset of $M$, then $A:=\bigcup_{p \in P} B p$ is $B$-absorbing.

Proof. It is trivial that $A$ is $B$-balanced because $B$ is multiplicatively idempotent. Suppose then that $P$ is $\mathcal{R}$-maximal. Fix an arbitrary $m \in M$. If $m \in P$, then $B m \subseteq A$. If $m \notin P$, then the maximality of $P$ implies that there exist $p \in P$ and $u \in \mathcal{U}(R)$ satisfying that $m=u p$. Since $B$ is left-feasible and $B u^{-1}$ is a 0-neighborhood of $R$, there exists $v \in \mathcal{U}(R)$ with $v B \subseteq B u^{-1}$. Then $v B m \subseteq B u^{-1} m=B p \subseteq A$ and hence $B m \subseteq v^{-1} A$. All these show that $A$ is $B$-absorbing.

Theorem 15. Let $R$ be an integral domain topological ring such that $\mathcal{U}(R)$ is open. Let $M$ be a torsionfree topological $R$-module. Consider a closed-unit zero-neighborhood $B \subseteq R$ verifying that $1 \notin \operatorname{int}(B)$ and $B \backslash\{0\} \subseteq \mathcal{U}(R)$. If $P \subseteq M$ is $\mathcal{R}$-free and $0 \in \operatorname{cl}(P \backslash\{0\})$, then $A:=\bigcup_{p \in P} B p$ is not a neighborhood of 0 . 
Proof. Suppose on the contrary that $A$ is a neighborhood of 0 . Then there exists $p_{0} \in P_{0} \cap \operatorname{int}(A)$ with $p_{0} \neq 0$. In view of Proposition 3 together with Theorem 10, we have that $M$ satisfies the strong open property. Then there exists $s \in \mathcal{U}(R) \backslash B$ such that $s p_{0} \in \operatorname{int}(A) \subseteq A=\bigcup_{p \in P} B p$. Since $P$ is $\mathcal{R}$-free and $B \backslash\{0\} \subseteq \mathcal{U}(R)$, the only possibility is that $s p_{0} \in B p_{0}$, i.e., there is $b \in B$ with $s p_{0}=b p_{0}$. Now, by taking into account that $R$ is an integral domain and $M$ is torsionfree, we conclude that $s=b \in B$, which is a contradiction.

Combining together Lemma 8 and Theorem 15, we obtain the desired result.

Corollary 8. Let $R$ be an integral domain topological ring such that $\mathcal{U}(R)$ is open. Let $M$ be a torsionfree topological $R$-module. Consider a left-feasible closed-unit zero-neighborhood $B \subseteq R$ verifying that $1 \notin \operatorname{int}(B)$ and $B \backslash\{0\} \subseteq \mathcal{U}(R)$. If $P \subseteq M$ is a maximal $\mathcal{R}$-free subset of $M$ and $0 \in \mathrm{cl}(P \backslash\{0\})$, then $A:=\bigcup_{p \in P} B p$ is $B$-balanced and B-absorbing but not a neighborhood of 0 .

Notice that the only restrictive hypothesis of Corollary 8 is the existence of an $\mathcal{R}$-free $P \subseteq M$ such that $0 \in \mathrm{cl}(P \backslash\{0\})$. The end of this section is devoted to find examples of $\mathcal{R}$-free subsets $P \subseteq M$ satisfying that $0 \in \mathrm{cl}(P \backslash\{0\})$.

Definition 7. We will say that a topological module $M$ over a topological ring $R$ is large if there exists a basis $\mathcal{B}$ of 0 -neighborhoods such that $\operatorname{card}(\mathcal{B}) \leq \operatorname{card}\left(\frac{M}{\mathcal{R}} \backslash\left\{[0]_{\mathcal{R}}\right\}\right)$.

Theorem 16. Let $R$ be a topological ring and $M$ a topological $R$-module. If $R$ is practical and $M$ is large, then there exists a maximal $\mathcal{R}$-free $Q \subseteq M$ such that $0 \in \operatorname{cl}(Q \backslash\{0\})$.

Proof. In view of Lemma 7, there exists a maximal $\mathcal{R}$-free $P \subseteq M$. Observe that by maximality, $P$ contains exactly one element of each equivalent class $[m]_{\mathcal{R}}$. In other words, $\operatorname{card}(P)=\operatorname{card}\left(\frac{M}{\mathcal{R}}\right)$ and hence $\operatorname{card}(P \backslash\{0\})=\operatorname{card}\left(\frac{M}{\mathcal{R}} \backslash\left\{[0]_{\mathcal{R}}\right\}\right)$. By hypothesis, there exists a basis $\mathcal{B}$ of neighborhoods of 0 such that $\operatorname{card}(\mathcal{B}) \leq \operatorname{card}\left(\frac{M}{\mathcal{R}} \backslash\left\{[0]_{\mathcal{R}}\right\}\right)=\operatorname{card}(P \backslash\{0\})$. Then we can take a one-to-one function $f: \mathcal{B} \rightarrow P \backslash\{0\}$. Since $R$ is practical by hypothesis, for every $U \in \mathcal{B}$ there exists an invertible $u_{U} \in \mathcal{U}(R)$ verifying that $u_{U} f(U) \in \operatorname{int}(U)$. Consider now $Q:=\left\{u_{U} f(U): U \in \mathcal{B}\right\} \cup P \backslash f(\mathcal{B})$. It is clear that $Q$ is a maximal $\mathcal{R}$-free subset of $M$ satisfying that $0 \in \mathrm{cl}(Q \backslash\{0\})$.

An example of a large module follows to conclude this final section and the manuscript.

Example 9. Let $R$ be a topological ring and $M$ a topological $R$-module. If $M$ is infinitely generated and first countable, then $M$ is large.

\section{Discussion}

The regularity of closed balls and open balls in seminormed modules has been proved to be mostly dependent on the properties of the underlying ring. Two of the ring properties involved in this manuscript are the open property and the practical property. One of our future lines of research is to study the relations between these two properties. It seems that they are independent properties.

On the other hand, it seems interesting to study more properties about internally $B$-balanced sets. In ([16] (Definition 2.1)), B-balanced sets are defined, and their properties are studied. In the next future, we pretend to unveil the implications between these two concepts.

Observe that Examples 6 and 7 show the existence of normed modules for which there exists a nonopen continuous linear functional. Indeed, since $R:=\mathbb{Q}[\pi]$ is an absolutely valued ring, then $M:=R \times R$ with the product topology is a normed module over $R$ (it only suffices to take the sup-norm, i.e., $\|(r, s)\|_{\infty}:=\max \{|r|,|s|\}$, which clearly induces the product topology on $R \times R$ ). Therefore, not even the existence of a module norm can guarantee the openness of all continuous linear 
functionals. However, Lemma 3 suggests that the existence of plenty of invertibles assures that more continuous linear functionals be open.

Finally, it is of a great interest to study the possible connections between topological modules and Molecular Topology [30]. At a first glance, it seems easier to tackle the problem of extending the classical Differential Manifold Theory to the topological module setting, which may be a key step before focusing into Molecular Topology. For instance, if $R$ is a topological ring, then one can define a topological $R$-manifold as a topological space $X$ in which every point has a neighborhood homeomorphic to a topological $R$-module. If $M$ is a previously fixed topological $R$-module, then we can define an $M$-topological manifold as a topological $R$-manifold in which every point has a neighborhood homeomorphic to $M$. This topic will become one of our future research lines.

Funding: This research was funded by Ministry of Science, Innovation and Universities of Spain, grant number PGC-101514-B-100; and by the 2014-2020 ERDF Operational Programme and by the Department of Economy, Knowledge, Business and University of the Regional Government of Andalusia, grant number FEDER-UCA18-105867.

Acknowledgments: The author would like to express his deepest gratitude towards Bellido for her valuable support and encouragement.

Conflicts of Interest: The author declares no conflict of interest. The funders had no role in the design of the study; in the collection, analyses, or interpretation of data; in the writing of the manuscript, or in the decision to publish the results.

\section{References}

1. Lefschetz, S. The role of algebra in topology. Bull. Amer. Math. Soc. 1937, 43, 345-359. [CrossRef]

2. Agarwal, R.P.; Gala, S.; Ragusa, M.A. A Regularity Criterion in Weak Spaces to Boussinesq Equations. Mathematics 2020, 8, 920. [CrossRef]

3. Bourbaki, N. Topological Vector Spaces. Chapters 1-5; Elements of Mathematics (Berlin); Springer: Berlin, Germany, 1987. [CrossRef]

4. Ragusa, M.; Razani, A. Weak solutions for a system of quasilinear elliptic equations. Contrib. Math. 2020, 1, 11-16.

5. Köthe, G. Topological Vector Spaces I; Translated from the German by D. J. H. Garling. Die Grundlehren der mathematischen Wissenschaften, Band 159; Springer: New York, NY, USA, 1969.

6. Beckenstein, E.; Narici, L.; Warner, S. Three-space problems in commutative algebras. Arch. Math. (Basel) 1983, 41, 447-453. [CrossRef]

7. Warner, S. Sheltered modules and rings. Proc. Am. Math. Soc. 1971, 30, 8-14. [CrossRef]

8. Warner, S. Linearly compact rings and modules. Math. Ann. 1972, 197, 29-43. [CrossRef]

9. Eisele, K.T.; Taieb, S. Weak topologies for modules over rings of bounded random variables. J. Math. Anal. Appl. 2015, 421, 1334-1357. [CrossRef]

10. Warner, S. Topological Fields; North-Holland Mathematics Studies; Notas de Matemática [Mathematical Notes], 126; North-Holland Publishing Co.: Amsterdam, The Netherlands, 1989; Volume 157.

11. Warner, S. Topological Rings; North-Holland Mathematics Studies; North-Holland Publishing Co.: Amsterdam, The Netherlands, 1993; Volume 178.

12. Arnautov, V.I.; Glavatsky, S.T.; Mikhalev, A.V. Introduction to the Theory of Topological Rings and Modules; Monographs and Textbooks in Pure and Applied Mathematics; Marcel Dekker, Inc.: New York, NY, USA, 1996; Volume 197.

13. Sakai, S. $C^{*}$-Algebras and $W^{*}$-Algebras, 1971; Classics in Mathematics; Springer: Berlin, Germany, 1998. [CrossRef]

14. García-Pacheco, F.J.; Naranjo-Guerra, E. Balanced and absorbing subsets with empty interior. Adv. Geom. 2016, 16, 477-480. [CrossRef]

15. García-Pacheco, F.J.; Naranjo-Guerra, E. A family of balanced and absorbing sets with empty interior. J. Nonlinear Func. Anal. 2017, 2017, 1-10. [CrossRef]

16. García-Pacheco, F.J.; Piniella, P. Geometry of balanced and absorbing subsets of topological modules. J. Algebra Appl. 2019, 18, 1950119. [CrossRef] 
17. García-Pacheco, F.J.; Piniella, P. Unit neighborhoods in topological rings. Banach J. Math. Anal. 2015, 9, 234-242. [CrossRef]

18. García-Pacheco, F.J.; Piniella, P. Linear topologies and sequential compactness in topological modules. Quaest. Math. 2017, 40, 897-908. [CrossRef]

19. Nowak, P.W. Group actions on Banach spaces. In Handbook of Group Actions. Volume II; International Press: Somerville, MA, USA, 2015; Volume 32, pp. 121-149.

20. Shulman, T. On subspaces of invariant vectors. Stud. Math. 2017, 236, 1-11. [CrossRef]

21. García-Pacheco, F.J. Complementation of the subspace of G-invariant vectors. J. Algebra Appl. 2017, 16, 1750124. [CrossRef]

22. García-Pacheco, F.J.; Pérez-Fernández, F.J. Pre-Schauder Bases in Topological Vector Spaces. Symmetry 2019, 11, 1026. [CrossRef]

23. García-Pacheco, F.J.; Sáez-Martínez, S. Normalizing rings. Banach J. Math. Anal. 2020. [CrossRef]

24. Steen, S.W.P. Introduction to the theory of operators IV Linear functionals. Proc. Camb. Philos. Soc. 1939, 35, 562-578. [CrossRef]

25. García-Pacheco, F.J.; Murillo-Arcila, M.; Miralles-Montolío, A. The spectral decomposition in unitary algebras. J. Math. Anal. Appl. 2019, submitted.

26. García-Pacheco, F.J. The finest locally convex module topology. Cambridge J. Math. 2020, submitted.

27. Loebl, R.I.; Paulsen, V.I. Some remarks on $C^{*}$-convexity. Linear Algebra Appl. 1981, 35, 63-78. [CrossRef]

28. Megginson, R.E. An Introduction to Banach Space Theory; Graduate Texts in Mathematics; Springer: New York, NY, USA, 1998; Volume 183. [CrossRef]

29. Piniella, P. Existence of non-trivial complex unit neighborhoods. Carpathian J. Math. 2017, 33, 107-114.

30. Diudea, M.V.; Gutman, I.; Jäntschi, L. Molecular Topology; Nova Science Publishers Inc.: Hauppauge, NY, USA, 2001.

(C) 2020 by the authors. Licensee MDPI, Basel, Switzerland. This article is an open access article distributed under the terms and conditions of the Creative Commons Attribution (CC BY) license (http://creativecommons.org/licenses/by/4.0/). 